\title{
Title: Mechanisms underlying cellular responses of cells from haemopoietic tissue to low dose/low LET radiation
}

Dr. Munira Kadhim ${ }^{1,2}$

1- Radiation and Genome Stability Unit MRC, Harwell, Didcot, Oxon, OX11 ORD, UK

Tel: +44 1235 834393, FAX: +44 1235 834776, email: m.kadhim@har.mrc.

2-Current address: School of Life Sciences, Oxford Brookes University, Oxford, OX3 OBP, UK

Email: mkadhim@brookes.ac.uk

\section{RELEVANCE STATEMENT}

To accurately define the risks associated with human exposure to relevant environmental doses of low LET ionizing radiation, it is necessary to completely understand the biological effects at very low doses (i.e., less than $0.1 \mathrm{~Gy}$ ), including the lowest possible dose, that of a single electron track traversal. At such low doses, a range of studies have shown responses in biological systems which are not related to the direct interaction of radiation tracks with DNA. The role of these "nontargeted" responses in critical tissues is poorly understood and little is known regarding the underlying mechanisms. Although critical for dosimetry and risk assessment, the role of individual genetic susceptibility in radiation risk is not satisfactorily defined at present.

The aim of the proposed grant is to critically evaluate radiation-induced genomic instability and bystander responses in key stem cell populations from haemopoietic tissue. Using stem cells from two mouse strains (CBA/H and C57BL/6J) known to differ in their susceptibility to radiation effects, we plan to carefully dissect the role of genetic predisposition on two non-targeted radiation responses in these models; the bystander effect and genomic instability, which we believe are closely related. We will specifically focus on the effects of low doses of low LET radiation, down to doses approaching a single electron traversal. Using conventional X-ray and $\mathrm{Y}$-ray sources, novel dish separation and targeted irradiation approaches, we will be able to assess the role of genetic variation under various bystander conditions at doses down to a few electron tracks. Irradiations will be carried out using facilities in routine operation for bystander targeted studies. Mechanistic studies of instability and the bystander response in different cell lineages will focus initially on the role of cytokines which have been shown to be involved in bystander signaling and the initiation of instability. These studies also aim to uncover protein mediators of the bystander responses using advanced proteomic screening of factors released from irradiated, bystander and unstable cells. Integral to these studies will be an assessment of the role of genetic susceptibility in these responses, using $\mathrm{CBA} / \mathrm{H}$ and $\mathrm{C} 57 \mathrm{BL} / 6 \mathrm{~J}$ mice. The relevance of in vivo interactions between stem cells and the stem cell niche will be explored in the future by re-implantation techniques of previously irradiated cells.

The above studies will provide fundamental mechanistic information relating genetic predisposition to important low dose phenomena, and will aid in the development of Department of Energy policy, as well as radiation risk policy for the public and the workplace. We believe the proposed studies accurately reflect the goals of the DOE low dose program. 


\section{PROJECT DESCRIPTION}

Here, we are providing a report on the work that was conducted at the Medical Research Council (MRC) for the above project during the period " 1 st March $2006-29^{\text {th }}$ February 2008". A poster from this work was presented at the UK-ARR meting in Belfast, April, 2007.

\section{Introduction}

There is strong evidence for genetic basis of susceptibility to radiation-induced genomic instability in both human and mouse cells (1). Differences in radio-sensitivity in response to low doses of high LET and high doses of low LET radiation are known between several mouse strains. However, it is vitally important to study the biological effects at very low doses of low LET ionizing radiation to better understand the risk associated with human exposure to relevant environmental doses of radiation.

Several evidences suggest that the cellular response to ionizing radiation involves the recognition of the radiation-induced damage and the initiation of signal transduction cascade(s), which lead to cell cycle arrest, damage repair, and a decision to survive, or die by apoptosis. Of all the types of DNA damage, DNA double strand break (DSB) is the greatest threat to the integrity of the genome. Important progress has been made in elucidating the DNA DSB checkpoint pathway. This pathway is constituted of sensors which recognize the presence of DNA DSBs, signal transducers which amplify the DNA damage signal and effectors which induce cell cycle delay and programmed cell death. The earliest signaling molecules, which are known to initiate the transduction cascade at damage sites, are protein kinases ataxia telangiectasia mutated (ATM) and ATM- and Rad-3-related (ATR). At the molecular level, activation of the DNA damage checkpoints is based on a phosphorylation cascade that starts with the activation of ATM and ATR, and members of the PIKK (phosphatidyl-inositol 3 kinase like protein kinase) family at the site of lesions (2).

In the current study two such common inbred strains, CBA/CaH and C57BL/6J, were selected to examine their sensitivity to the lowest possible dose ( $<0.1 \mathrm{~Gy}$ ) of low LET X-rays. Therefore, an analysis of the expression and activation of ATM, ATR and their downstream target genes (effectors) in $\mathrm{CBA} / \mathrm{CaH}$ and $\mathrm{C57BL} / 6 \mathrm{~J}$ haemopoietic stem cell, with and without exposure to ionising radiation (Low Let X-rays), could help to understand the role of the different genetic susceptibility observed in these two strain of mice.

\section{Material and Methods}

CBA/CaH and C57BL/6J in-bred strains of mouse bred at the MRC were used. In addition to the data previously obtained from the $\mathrm{CBA} / \mathrm{CaH}$ strain, results presented here are those obtained from the C57BL/6J strain. As shown in Figure 1, femoral bone marrow cells were isolated from 10-12 week old male CBA/CaH and C57BL/6J mice. The cells were exposed to $0,0.01,0.05,0.1$ and 3 Gy X-rays (250 keV). Immediately after irradiation/sham-irradiation, cells were cultured in $25 \mathrm{~cm}^{2}$ Falcon culture flasks containing modified $\alpha$ Eagles media supplemented with $25 \%$ horse sera, antibiotics and sources of pre-tested conditioned media (3) to permit measurement of GI by chromosomal analysis 2 and 15 population doublings ( $\mathrm{pd}$ ) post-irradiation. Additionally, the clonogenic CFU-A assay (3) was used to obtain clones of cells from members of the 
haemopoietic stem cell compartment. Survival colonies were assessed and harvested 6 and 7 days post-irradiation for clonal chromosome instability analysis.

Whole cell extracts were analysed for the background expression and activation of ATM and ATR; immediately, 2 and 24 hours post-irradiation. Cells were lysed at a concentration of approximately $1 \times 10^{7}$ cells $/ \mathrm{ml}$ in cell lysis buffer $(20 \mathrm{mM}$ HEPES, pH 7.20, $50 \mathrm{mM} \mathrm{NaCl}, 10 \mathrm{mM}$ EDTA, 2 mM EGTA, 0.5 nonidet P-40 (NP-40), $0.5 \mathrm{mM}$ PMSF, $10 \mu \mathrm{g} / \mathrm{ml}$ Leupeptine, $0.5 \mathrm{mM}$ DTT, $10 \mathrm{mM} \mathrm{Na2MoO} 4 \cdot \mathrm{H} 2 \mathrm{O}, 10 \mathrm{mM}$ Na3VO4, 100mM NaF) to eliminate the red blood cell population. For immunoblot analysis, lysates were first adjusted to contain equal amounts of proteins $(20 \mu \mathrm{g}$ total proteins) using the Bradford assay and were then dissolved in SDS-PAGE sample buffer prior to separation by $7.5 \%$ SDS polyacrylamide gel. Analysis was performed with ECL detection kit (Amersham). The following antibodies were used: mouse ATM and ATR (Santa Cruz Biotechnology, Inc) diluted 1:200.

\section{Cells cultured in T175}

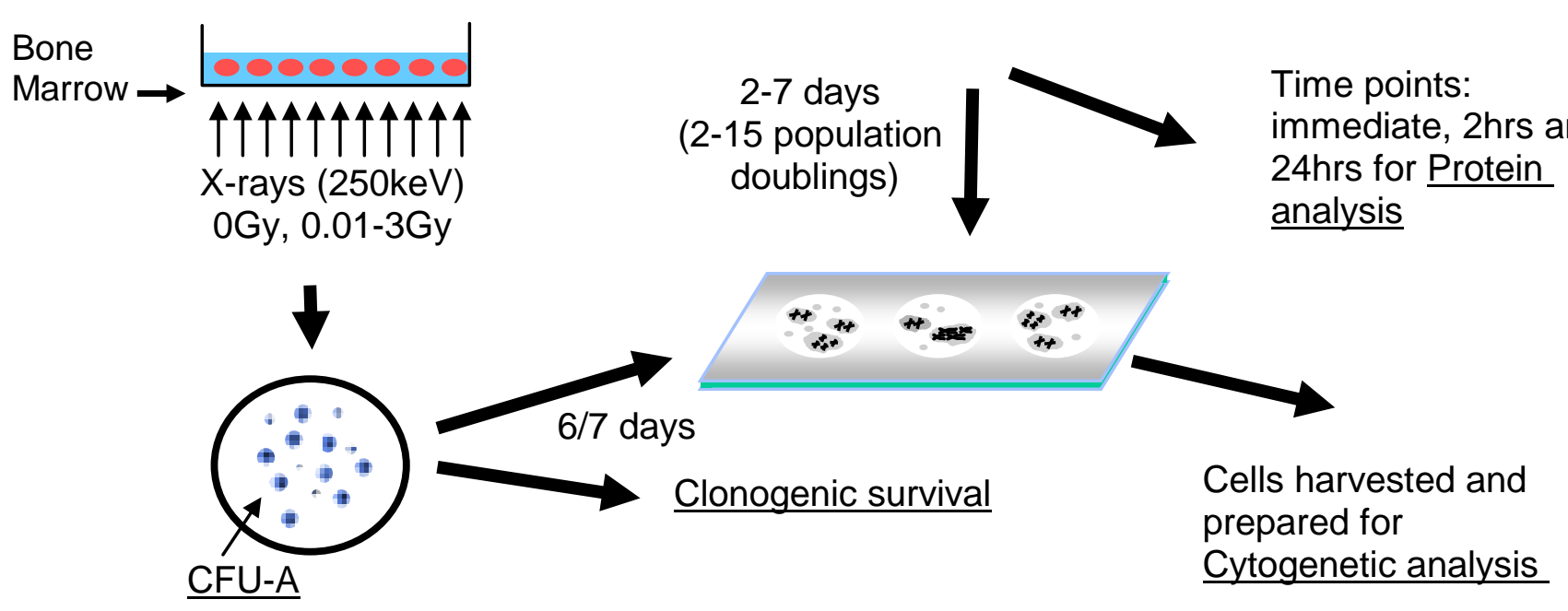

Figure 1. Schematic diagram for the methods and endpoints utilized. 


\section{Results}

Clonogenic survival- Figure 2a

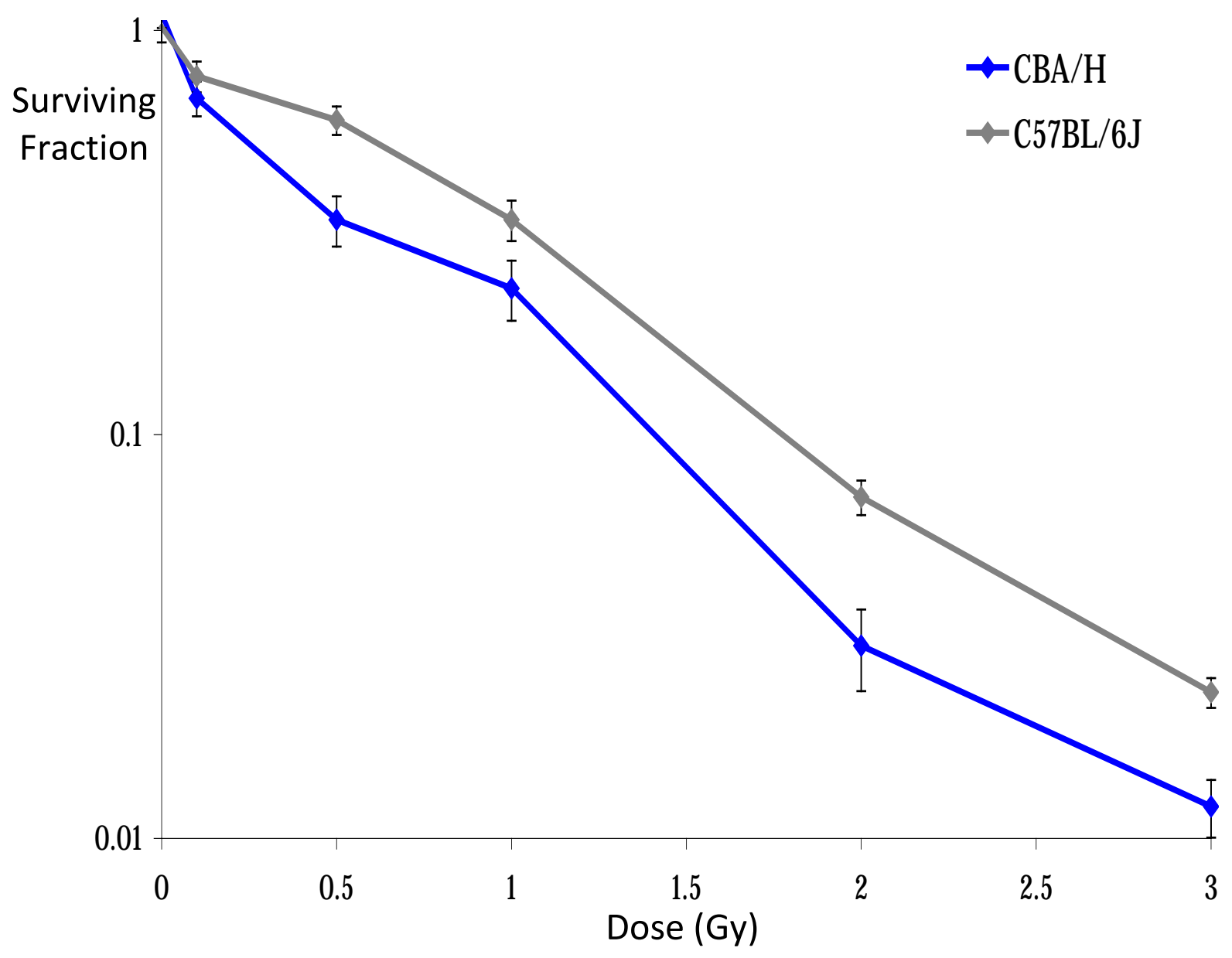


$\underline{\text { Target cell number- Figure } 2 \mathrm{~b}}$

\begin{tabular}{|c|c|c|c|}
\cline { 2 - 3 } \multicolumn{1}{c|}{} & \multicolumn{2}{c|}{ Mouse Strain } & \multicolumn{1}{c}{} \\
\hline Stem cells & $\mathrm{CBA} / \mathrm{H}$ & C57BL/6J & Fold difference \\
\hline CD34+ & $0.27 \%$ & $1.23 \%$ & 4.6 \\
\hline LY6+ & $0.07 \%$ & $0.50 \%$ & 7.1 \\
\hline CD34+/LY6+ & $0.08 \%$ & $0.15 \%$ & 1.9 \\
\hline
\end{tabular}

Overall, survival was significantly reduced in CBA/CaH compared to C57BL/6J (see Figure 2a) after low LET X-rays ( $p=0.013)$. Surviving fractions are corrected to respective control values. Error bars represent $+/$ - SEM of 3 experiments. Potential target cell number are $2-7 \times$ greater in C57BL/6J compared to CBA (Figure 2b).

Chromosome instability analysis- Figure 3
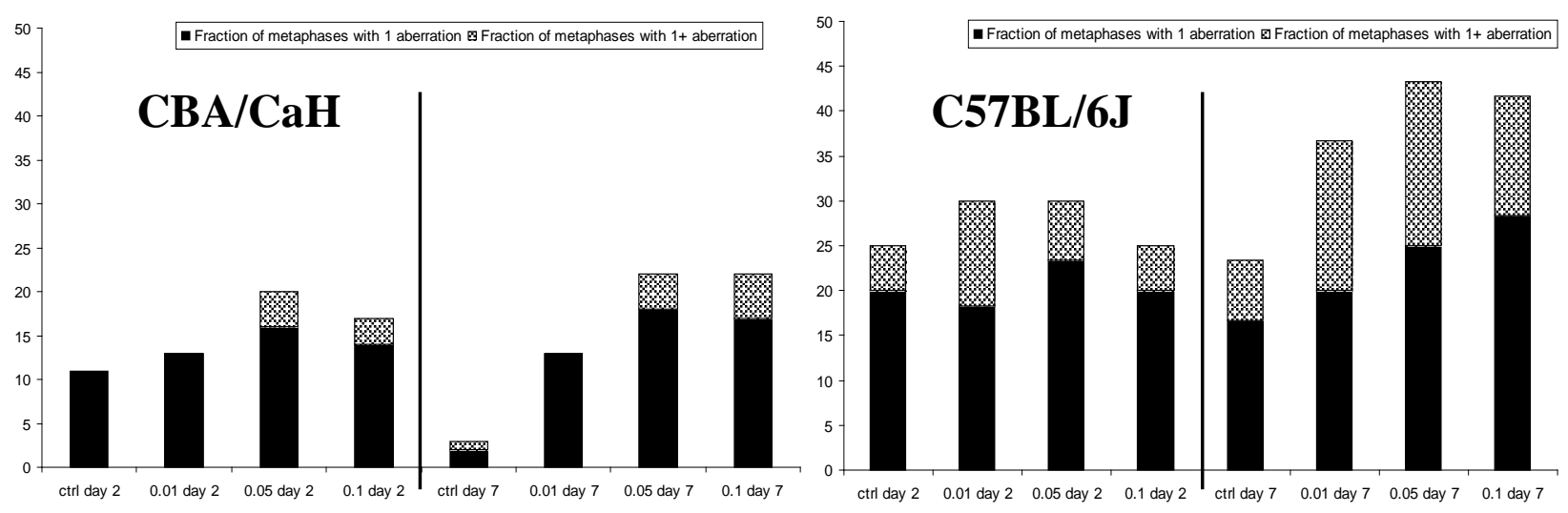
Very low doses of low LET radiation induce chromosome/genetic instability in CBA/CaH and C57BL/6J mouse haemopoietic stem cells (figure 3)

Chromosome instability was induced with very low doses of low LET X-rays in stem cells derived from $\mathrm{CBA} / \mathrm{CaH}$ and $\mathrm{C} 57 \mathrm{BL} / 6 \mathrm{~J}$ mice, but the magnitude of induction demonstrated some strain specificity. The level of instability in $\mathrm{CBA} / \mathrm{CaH}$ mouse strain at 2 and $15 \mathrm{pd}$ post irradiation were similar following $0.01 \mathrm{~Gy}$ exposure with a significant increase after exposure to 0.05 and $0.1 \mathrm{~Gy}$. In contrast, the chromosomal instability in C57BL/6J was demonstrated to be notably higher at 15 pd post-irradiation compared to the initial damage with no obvious dose dependency. The percentage of heavily damaged cells was greater in C57BL/6J than in CBA/CaH. A large fraction of the difference may be due to the contribution of heavily damaged cells.

Western Blot analysis (Figures 4a\&4b)

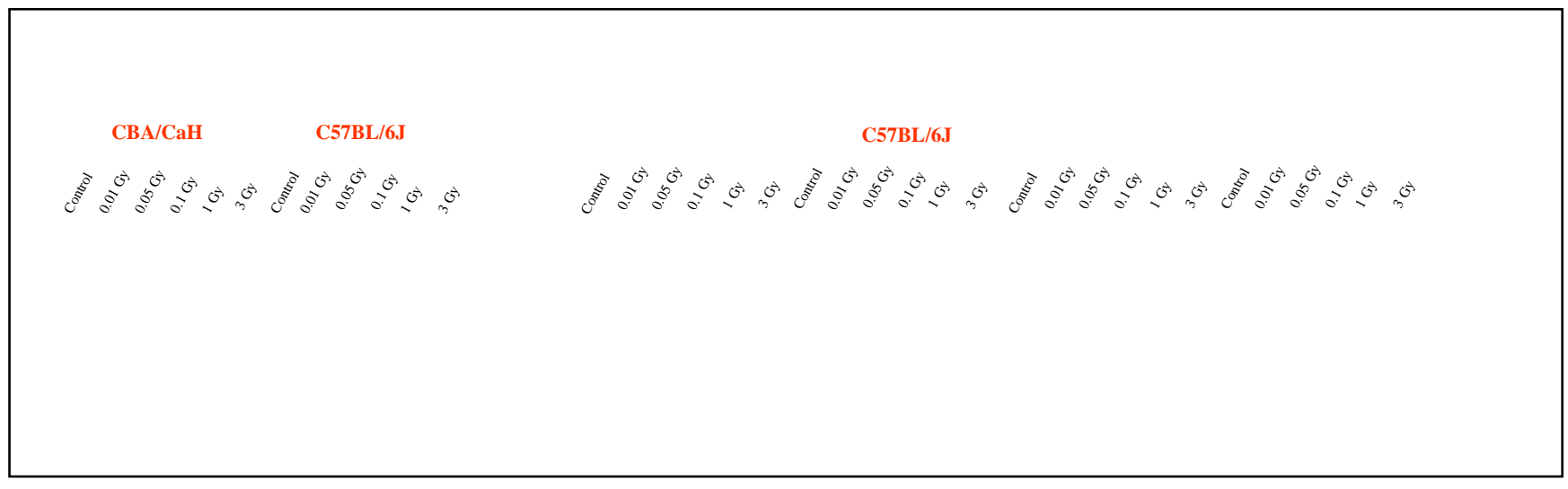




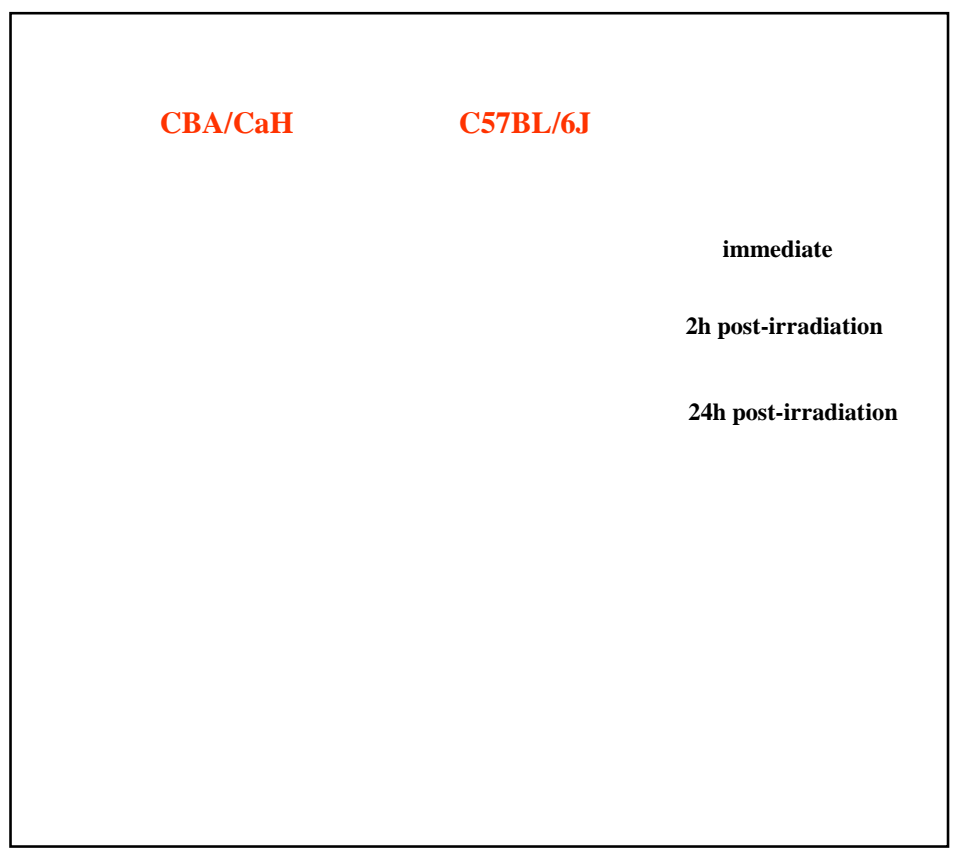




\section{Discussion}

The current study demonstrates that chromosomal instability was induced in $\mathrm{CBA} / \mathrm{CaH}$ and C57BL/6J strains of mice following very low doses of low LET irradiation (< $0.1 \mathrm{~Gy}$ ). Although instability was significantly induced in cultures obtained from CBA/CaH and C57BL/6J at 2 and 15 population doublings, after very low doses $(<0.1 \mathrm{~Gy})$, we also observed qualitative differences relating to more heavily damaged cells after exposure to $0.05 \mathrm{~Gy}$ and $0.1 \mathrm{~Gy}$ than $0.01 \mathrm{~Gy}$ in both strains (Fig. 1)

Phosphorylated ATM in both $\mathrm{CBA} / \mathrm{CaH}$ and $\mathrm{C} 57 \mathrm{BL} / 6 \mathrm{~J}$ showed an active pathway in these cells. However, following exposure to low dose X-ray, ATM was significantly more active in CBA/CaH than in C57BL/6J. To support these differences in ATM activity observed in both strains of mice, the first ATM downstream target gene Chk2 was investigated. Chk2 activity results were concordant with the ATM phosphorylation trend observed and demonstrated that ATM is required for the phosphorylation of Chk2 kinase. These data suggest that C57BL/6J mice are less effective in repairing the damage caused by ionizing radiation and support cytogenetic analysis which showed a greater percentage of heavily damaged cells in C57BL/6J compared to CBA/CaH.

The defective ATM activity in C57BL/6J erroneously repairs the damage, leading to decreased apoptosis (especially at high doses of ionizing radiation and by Bax down-regulation), increased survival and chromosomal instability. Collectively, these findings suggest a model wherein normal recognition and repair of DNA damage leads to cell death and a consequent reduction in aberrant cells especially with more than one aberration (e.g. CBA/CaH.) Conversely, normal recognition of damage but abnormal repair might lead to increased survival, decreased apoptosis and increased aberrant metaphases with more than one aberration (e.g. C57BL/6J).

\section{References}

1. Kadhim, M. A. (2003). Role of genetic background in induced instability. Oncogene 22 (45): 6994-6999

2. Abraham, R.T. (2004). PI 3-kinase related kinases: 'big' players in stress-induced signalling pathways. DNA Repair (Amst.) 3: 883-887

3. Lorimore S. A., Pragnell I. B., Eckmann L. and Wright E. G. (1990). Synergistic interactions allow colony formation in vitro by murine haematopoietic stem cells. Leukemia Research 14: 481-489 